Mens

revue d'histoire intellectuelle de l'Amérique française

\title{
Andrée Lajoie. Vive la recherche libre! Les subventions publiques à la recherche en sciences humaines et sociales au Québec, Montréal, Lilber, 2009, 224 p.
}

\section{Marcel Martel}

Volume 10, numéro 2, printemps 2010

URI : https://id.erudit.org/iderudit/1023313ar

DOI : https://doi.org/10.7202/1023313ar

Aller au sommaire du numéro

Éditeur(s)

Centre de recherche en civilisation canadienne-française

ISSN

1492-8647 (imprimé)

1927-9299 (numérique)

Découvrir la revue

Citer ce compte rendu

Martel, M. (2010). Compte rendu de [Andrée Lajoie. Vive la recherche libre! Les subventions publiques à la recherche en sciences humaines et sociales au Québec, Montréal, Liber, 2009, 224 p.] Mens, 10(2), 114-116.

https://doi.org/10.7202/1023313ar d'utilisation que vous pouvez consulter en ligne.

https://apropos.erudit.org/fr/usagers/politique-dutilisation/ 


\section{Andrée Lajoie. Vive la recherche libre! Les subventions publiques à la recherche en sciences humaines et sociales au Québec, Montréal, Liber, 2009, 224 p.}

Voici un livre qui intéressera ceux qui ont obtenu une subvention de recherche d'un organisme gouvernemental ou qui songent à soumettre une telle demande. Il faut féliciter Andrée Lajoie, chercheuse chevronnée et observatrice de l'évolution des programmes offerts par les organismes subventionnaires, pour la publication de son ouvrage. L'auteure a utilisé une partie de l'argent qu' elle a reçu en obtenant la médaille d'or du Conseil de recherches en sciences humaines du Canada pour étudier l'évolution de la recherche subventionnée par les gouvernements fédéral et québécois.

L'ouvrage traite du thème du rôle de l'État dans le financement de la recherche universitaire, sujet sur lequel bien peu d'universitaires ont écrit. L'État doit-il orienter cette recherche en privilégiant des sujets précis ou, au contraire, laisser la créativité des chercheurs s'exprimer en ne déterminant aucun sujet de recherche? Certes, on ne peut ignorer les critiques de certains individus, souvent à l'extérieur du milieu universitaire, sur le caractère pointu de certaines recherches subventionnées. Cela reflète le courant néolibéral axé sur la productivité, l'efficacité et l'utilité à court terme de la recherche. Par ailleurs, ce mantra révèle l'ignorance sur la manière dont s'effectue la recherche scientifique, que ce soit dans les domaines de la médecine, de la physique, du droit ou de l'histoire. La recherche prend du temps et requiert des ressources humaines et matérielles. Ce qui peut paraitre comme un sujet frivole et peu utile s'avère souvent fort pertinent lorsque la recherche est complétée.

Le livre se divise en deux parties. La première traite de l'évolution des programmes de subvention fédéraux et québécois. Bien que l'auteure traite rapidement de la création de ces programmes, elle montre de manière convaincante les effets structurants de ces subventions sur la recherche. Andrée Lajoie constate un déclin progressif de l'appui du gouvernement fédéral, qui intervient dans un champ de compétence provinciale rappelle-t-elle, à la recherche individuelle 
dite libre. En même temps, l'État fédéral crée des programmes ciblés et d'autres favorisant le développement de partenariats avec des groupes à l'extérieur du milieu universitaire. Du côté de l'État québécois, les programmes se distinguent, dès leur création dans les années 1970, car ceux-ci encouragent la recherche ciblée et concertée. Ils favorisent également la formation d'équipes pour mener à terme des projets de recherche.

Dans la seconde partie, l'auteure présente les résultats de son enquête auprès de cinquante individus sur leur perception des organismes subventionnaires québécois et fédéraux. Ce groupe inclut quarante professeurs qui ont obtenu des subventions, et dix administrateurs universitaires ou individus rattachés aux organismes subventionnaires. La plupart des participants à l'enquête œuvrent dans sept universités québécoises et proviennent de cinq disciplines, soit histoire, sociologie, droit, science politique et philosophie. Même si les participants à l'enquête admettent que la recherche se porte relativement bien au Québec, plusieurs professeurs interrogés dénoncent le caractère " utilitariste de la recherche " et la nécessité de former des équipes de recherche pour obtenir des subventions. Sur ce dernier point, certains participants à l'enquête relèvent le fait que bien de ces équipes n'ont que le nom, car en termes de fonctionnement, il y a très peu d'interactions entre les membres de l'équipe. Si certains professeurs mentionnent que l'obtention d'une subvention de recherche est maintenant requise pour obtenir la permanence et la promotion, la plupart dénoncent l'appui financier étatique qui profite surtout aux sciences et bien peu aux sciences humaines. Sur les questions des partenariats avec des organismes non universitaires, du rayonnement international de la recherche et des cibles de recherche déterminées par les organismes subventionnaires, les professeurs sont souvent plus critiques à l'égard de ces pratiques que les administrateurs qui ont participé à l'enquête.

Le dernier chapitre traite des facteurs sociostructurels qui influent sur l'obtention de subventions. L'auteure explore les questions relatives à la taille des universités, à leur localisation géographique, aux tâches 
administratives, souvent exigeantes pour les chercheurs qui débutent dans la profession, au genre et au soutien à la recherche. Sur ce dernier point, le soutien institutionnel à la recherche varie en fonction de la taille de ces institutions et de leur situation géographique. Dans le cas des universités situées en région, elles ont souvent peu ou pas de programmes d'études supérieures. Par conséquent, il est difficile pour le professeur subventionné d'embaucher des assistants de recherche. Par ailleurs, certains des professeurs interrogés reconnaissent les efforts d'appui à la recherche de certaines institutions.

Dans sa conclusion, l'auteure critique les efforts des organismes subventionnaires pour diriger la recherche. Elle propose des solutions, notamment l'augmentation du financement de l'État québécois au réseau universitaire et un accroissement des sommes attribuées par les organismes subventionnaires à la recherche dite libre. Sur ce dernier point, Andrée Lajoie suggère qu'au moins $50 \%$ des sommes attribuées par les organismes subventionnaires soient affectées à la recherche libre. Pour y parvenir, il faudra sabrer les programmes existants et rediriger les sommes vers la recherche individuelle.

Nos critiques sur cet ouvrage sont peu nombreuses. Ainsi, il aurait été souhaitable d'étudier les objectifs politiques des organismes subventionnaires, les pressions de la classe politique et d'autres groupes sur ces organismes ainsi que les rapports entre le développement de la pensée néolibérale et les orientations de ces organismes. Ainsi, quels facteurs ont amené une multiplication des programmes de subvention de recherche depuis les trente dernières années? Quel est le rôle des universitaires dans la création, la transformation et l'abandon de certains programmes de recherche?

Malgré ces quelques critiques, le livre d'Andrée Lajoie est pertinent et fort approprié. J'espère que d'autres chercheurs analyseront le rôle non seulement de l'État mais aussi du secteur privé dans le financement de la recherche universitaire.

- Marcel Martel 